\title{
Mesh Fixation in Laparoscopic Inguinal Hernia Repair: Absorbable vs. Non-Absorbable Tacks
}

\author{
Facundo Iriarte, Joaquín Fernández Alberti*, Raúl Eduardo Croceri, Pablo Medina, and Daniel Enrique Pirchi \\ Department of General Surgery, Hospital Británico de Buenos Aires, Perdriel 74, CABA, 1280, Argentina
}

*Corresponding author: Joaquín Fernández Alberti, Department of General Surgery, Hospital Británico de Buenos Aires, Perdriel 74, CABA, 1280, Argentina, Tel: 549-221-4375047; E-mail: joaquinfernandezalberti@gmail.com

Received: 10 Aug, 2020 | Accepted: 05 Sep, 2020 | Published: 11 Sep, 2020

Citation: Iriarte F, Alberti JF, Croceri RE, Medina P, Pirchi DE (2020) Mesh Fixation in Laparoscopic Inguinal Hernia Repair: Absorbable vs. Non-Absorbable Tacks. J Surg Open Access 6(6): dx.doi.org/10.16966/2470-0991.225

Copyright: (C) 2020 Iriarte $F$, et al. This is an open-access article distributed under the terms of the Creative Commons Attribution License, which permits unrestricted use, distribution, and reproduction in any medium, provided the original author and source are credited.

\begin{abstract}
Background: Laparoscopic inguinal hernia repair is an accepted technique for the treatment of primary inguinal hernias. There are several ways of mesh fixation including absorbable, non-absorbable staplers and even human fibrin glue. Little evidence is found when looking for a difference in recurrence rates between the first two.

Methods: A group of patients who were treated by primary unilateral or bilateral laparoscopic inguinal hernia repair (TAPP) between the years 2013 and 2014, including 24 months, were assessed with a total amount of 396 patients or 466 procedures. The group was divided in two, whether the mesh was fixated with non-absorbable staples (Group 1) or absorbable staples (Group 2). A minimum follow up of 2 years was required and performed by physical exam and telephone call.

Results: We had a minimum follow up of two years with $92 \%$ of success, with a total of 374 patients and 444 procedures assessed in the process. Group 1 had 274 procedures performed with non-absorbable staples (Protack ${ }^{\circledR}$ ), and Group 2170 procedures with absorbable staples (Securestrap ${ }^{\circledR}$ ). 4 recurrences were found, 3 of them were from Group $1(1.1 \%)$ and 1 from Group $2(0.59 \%) p=1, O R=0.53$ 95\% Cl=0.05- 5.1.

Conclusions: In mid-term follow up there were no statistically significant differences of recurrences among the two groups. Randomized studies with a larger number of cases are necessary to confirm these findings and draw more robust and objective conclusions.
\end{abstract}

Keywords: Absorbable; Laparoscopy; Non-absorbable; Mesh fixation; Inguinal hernia

\section{Introduction}

Since its description in the 1990s, Laparoscopic Inguinal Hernia Repair has gained a wider acceptance with increasing experience in the technique, technical advances, and the benefits that this approach offer to patients [1]. Nowadays, it has become as effective and equally recommended as Lichtenstein repair for the treatment of inguinal hernias in adults with similar or same results [2].

There are 3 different types of techniques commonly used, the Lichtenstein repair, Transabdominal Preperitoneal (TAPP), and Totally Extraperitoneal (TEP) approach. Common complications of these procedures are seroma, hematoma, chronic pain, and recurrence. Chronic pain is an important problem after an inguinal hernia repair, it is extremely uncomfortable for patients and affects their daily living. The most commonly involved nerves are the Genitofemoral nerve in open repair, and Lateral Femoral Cutaneous nerve in laparoscopic procedures [3]. Rates of the last one vary between $0.1 \%$ to $10 \%$ [4].

After the incorporation of mesh in the procedures the incidence of recurrences diminished to less than $2 \%$ in some specialized centers
[5], but it can be as high as 7.9\% [6]. In endoscopic procedures, recurrence mechanism have been thoroughly studied and the main ones are insufficient dissection, inadequate prosthetic overlap, improper fixation, slit in the mesh, small mesh size, folding or twisting of the prosthesis, missed hernias and lifting of the mesh secondary to hematoma formation $[7,8]$. A small mesh size is an independent risk factor for recurrence independently of the mesh type (heavy or lightweight) [7,9]. Also, recurrence after fixation and non-fixation has been studied, but, of several randomized controlled studies and case-control studies, only one found no significant differences in the incidence of recurrence between fixated and non-fixated repairs [9], and it is recommended that mesh fixation is carried out for defects $\geq 4 \mathrm{~cm}$ [9]. When it comes to differentiate whether there is any difference in recurrence rates regarding the use of different types of staples (absorbable and nonabsorbable) we found no studies on the matter.

The aim of this study was to differentiate recurrence rates between nonabsorbable (Protack ${ }^{\oplus}$ ) and absorbable (Securestrap ${ }^{\star}$ ) staples, in a high volume laparoscopic center. 


\section{Methods}

The present study included every person over 18 years old with primary, unilateral or bilateral inguinal hernia that were treated with TAPP laparoscopic inguinal hernia repair, during the years 2013 and 2014 at the British Hospital of Buenos Aires, Argentina.

For the present study all primary TAPP laparoscopic inguinal hernia repair, unilateral or bilateral, that were performed in the years 2013 and 2014 (24 months) in a single center, were analyzed.

Absorbable (Securestrap ${ }^{\star}$ ) and non-absorbable (Protack ${ }^{\circledR}$ ) staples were used for mesh fixation. The election of the staple use is made by the surgeon preference and the availability offered by health insurance companies. Two groups were established, the one that utilized non-absorbable staple (Group 1) and the one with absorbable staple (Group 2).

Inclusion criteria was every person over 18 years old, with unilateral or bilateral, primary inguinal hernia.

Exclusion criteria were recurrent inguinal hernia, Totally Extraperitoneal inguinal hernia repair, and laparoscopic hernioplasty performed with other types of mesh fixation, e.g. Fibrin glue sealant.

Patients were assessed by physical exam at 7 and 30 post-operative days, 6 months after, and a final physical exam at 2 years. Those that could not go to the hospital were assessed by a telephone call.

Demographic data (gender, age, BMI, and American Society of Anesthesiologist classification), surgical data (surgical time, mesh size) and from the inguinal hernia (type and size of hernia) were analyzed between the two groups. Post-operative data was directed to morbidities and recurrences.

All procedures and controls carried out on the patients were subject to their informed consent.

\section{Surgical technique}

In the TAPP repair a three-port technique was routinely employed under general anesthesia (at our center, an umbilical $10 \mathrm{~mm}$ trocar is placed and two $5 \mathrm{~mm}$ trocars in the left flank). The peritoneum was mobilized transabdominally above the hernial defect and meticulous blunt and sharp dissection was carried out to separate the adhesions from the old mesh and the surrounding structures. The anatomical landmarks (Cooper's ligament, the iliopubic tract, and the inferior epigastric vessels) were identified and the etiology and type of the recurrent hernia were determined. After adequate space was created around the cord structures, a new polypropylene mesh of approximately $15 \times 10 \mathrm{~cm}$ was placed over the old mesh and fixed with5tacks (absorbable or non-absorbable) distributed 2 on the Cooper's ligament, and 3 at the aponeurotic arch to reinforce the myopectineal orifice with special care on respecting the triangle of pain to avoid nerve injury. The peritoneum was closed with a running suture. Following desufflation, the trocar sites were closed. No drains were required.

\section{Statistical analysis}

Quantitative variables are expressed as means with standard deviation and range, while qualitative variables are described as percentages. Comparison studies of the two groups were performed using the Mann Whitney and Fisher tests, respectively. A $p<0.05$ was considered statistically significant. Graphpad Prism 8.0.2 software was used.

\section{Results}

A total of 396 patients were included in the study with 466 procedures. We had a follow up of patients at 2 years of $96 \%(22$ patients did not show up to the consult neither answered phone calls). The analysis was performed over 374 patients and 444 procedures. Group 1 had 274 procedures and Group 2, 170. 4 recurrences were found approximately after 1 year of the surgery, 3 in group $1(1.1 \%)$, and 1 in group $2(0.59 \%), p=1, O R=0.53,95 \%$ $\mathrm{CI}=0.05-5.1$ showing no statistically differences between both groups.

Variables such as gender, BMI, and inguinal hernia size, demonstrated no statistically differences between the groups (Table 1). Hernia size was grouped in $\mathrm{T} 1$ and $\mathrm{T} 2$ of Nyhus classification and T3 separately. Hernia size was distributed between groups as follow: for group 1, 90.5\% were $\mathrm{T} 1 / 2$ and $9.5 \% \mathrm{~T} 3$, vs. group 2 that presented $90.6 \%$ of $\mathrm{T} 1 / 2$ and $9.4 \% \mathrm{~T} 3, \mathrm{p}=0.9$ (Figure 1 ).

The percentage of patients ASA III was similar between the two groups, $8.1 \%$ in group 1 and $7.1 \%$ in group $2, \mathrm{p}=0.59$ (Figure 2).

Age on the other hand, was found to have a rate of $33.5 \%$ of patients older than 65 years old in group 1 and $24.1 \%$ in group 2, $\mathrm{p}=0.04$, $\mathrm{OR}=0.62$.

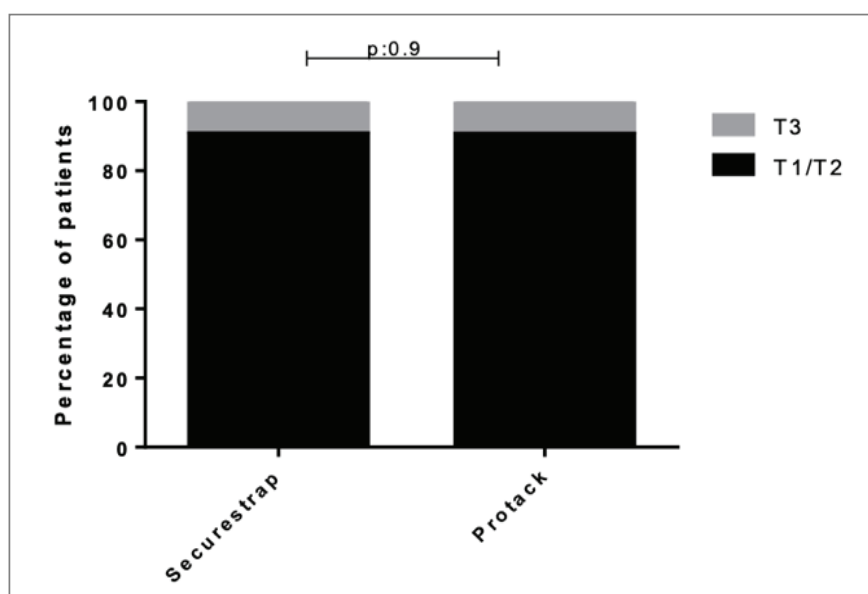

Figure 1: Hernia size distribution according the Nyhus classification.



Figure 2: Patient distribution according the American Society of Anesthesiologist classification. 
Table 1: Demographic data.

\begin{tabular}{|l|c|c|c|}
\hline & Protack & Secure strap & p \\
\hline $\mathrm{N}$ & 274 & 170 & \\
\hline Age & $55.3 \pm 15$ & $54.4 \pm 14.8$ & 0.5 \\
\hline Gender:n(percentage) & $248(90.5)$ & $154(90.5)$ & 0.9 \\
\hline BMI (kg/m²) & $26.3 \pm 3.7$ & $26.1 \pm 3.8$ & 0.54 \\
\hline Previous surgery:n (percentage) & $81(29.6)$ & $65(38.2)$ & 0.06 \\
\hline Recurrence:n (percentage) & $3(1)$ & $1(0.6)$ & 1 \\
\hline \multicolumn{4}{|c|}{ Coexisting Morbidities } \\
\hline Hypertension (percentage) & 25.2 & 35.8 & $<0.05$ \\
\hline Dyslipidemia (percentage) & 1.2 & 14.1 & 0.08 \\
\hline Smoking (percentage) & 7.6 & 8.2 & 0.85 \\
\hline Diabetes (percentage) & 3.6 & 4.1 & 0.8 \\
\hline
\end{tabular}

\section{Discussion}

The cause of hernia recurrences is multifactorial. This includes both technical and non-technical risk factors. This is a retrospective study that compared the incidence rate of recurrences in TAPP inguinal hernia repairs between absorbable and non-absorbable staples, in a single high volume laparoscopic center.

Among non-technical patient-related risk factors for recurrences there can be numbered age, gender, BMI, between others. In our study, gender, BMI, and inguinal hernia size were not statistically different between G1 and G2. Age on the other hand, was found to have a rate of $33.5 \%$ of patients older than 65 years old in G1 and $24.1 \%$ in $\mathrm{G} 2, \mathrm{p}=0.04, \mathrm{OR}=0.62$. Regarding this, there is a study that proof that this factor has no relevance in laparoscopic inguinal herniorrhaphy recurrences [10].

Regarding technical factors, mesh fixation is a controversial point in the literature. There are several ways to secure a mesh during a TAPP procedure which includes absorbable and non-absorbable tacks, fibrin glue, among others (2-4). It seems that no differences are found in recurrence rates between different methods like tackers $v s$ suture, tackers $v s$ glue and even there are some trials that demonstrate that no fixation of the mesh is safe [1,11-15]. On the other hand, based on the results of a multivariate analysis of 11.230 cases from a Herniamed registry study, a significant risk of recurrence is found not only in the group of non-fixation in case of direct hernias but also for combined hernias [16]. Furthermore, the European Hernia Society for the treatment of inguinal hernia in adults, published in 2014, it is stated that mesh fixation should be performed in large direct hernias [17]. All in all, nowadays still most surgeons prefer fixing the mesh in laparoscopic procedures in one way or another [6]. We believe that the most important risk factor for recurrence is the lack of experience of the treating surgeon and an inadequate surgical technique.

In this study, our aim was to compare recurrence rate between absorbable and non-absorbable staples given that most cases in our center are performed with a TAPP approach using these two fixing methods, and the scant, if there is, evidence in the literature in this specific matter. We found no statistically significant difference in recurrence rates between the groups, although there was a tendency to have more recurrences in the non-absorbable staple group.

Although the analysis of postoperative pain was not studied, there was no chronic postoperative pain recorded in the entire series under study. We believe that the possibility of developing chronic postoperative pain by nerve entrapment would not be directly related to the composition of the material, but to the correct anatomical knowledge of the inguinocrural region, and to avoid the placement of tackers at the level of the pain triangle.

This study contributes to the literature for those centers that still, by several reasons, use different staple options for TAPP laparoscopic inguinal hernia repair.

\section{Conclusions}

In mid-term follow up there were no statistically significant differences of recurrences among the two groups. Although it seems that there are no differences in recurrence rate between fixation methods, still this is employed by most surgeons and it is certainly indicated in several hernia laparoscopic repairs. Randomized studies with a larger number of cases are necessary to confirm these findings and draw more robust and objective conclusions.

\section{Conflict of Interests}

Drs. Joaquín Fernández Alberti, Facundo Iriarte, Raúl Eduardo Croceri, Pablo Medina and Daniel Enrique Pirchi have no conflicts of interest or financial ties to disclose. This research did not receive any specific grant from funding agencies in the public, commercial, or not-for-profit sectors.

\section{References}

1. Kleidari B, Mahmoudieh M, Yaribakht M, Homaei Z (2014) Mesh fixation in TAPP laparoscopic hernia repair: Introduction of a new method in a prospective randomized trial. Surg Endosc 28: 531-536.

2. Simons MP, Aufenacker T, Bay-Nielsen M, Bouillot JL, Campanelli G, et al. (2009) European Hernia Society guidelines on the treatment of inguinal hernia in adult patients. Hernia 13: 343-403.

3. Bueno J, Serralta A, Planells M, Rodero D (2004) Inguinodynia After Two Inguinal Herniorrhaphy Methods. Surg Laparosc Endosc Percutan Tech 14: 210-214.

4. Lovisetto F, Zonta S, Rota E, Mazzilli M, Bardone M, et al. (2007) Use of human fibrin glue (Tissucol) versus staples for mesh fixation in laparoscopic transabdominal preperitoneal hernioplasty: A prospective, randomized study. Ann Surg 245: 222-231.

5. Stoppa RE (1989) The treatment of complicated groin and incisional hernias. World J Surg 13: 545-554.

6. Siddaiah-Subramanya M, Ashrafi D, Memon B, Memon MA (2018) Causes of recurrence in laparoscopic inguinal hernia repair. Hernia 22: 975-986.

7. Lowham AS, Filipi CJ, Fitzgibbons RJ, Stoppa R, Wantz GE, et al. (2003) Mechanisms of hernia recurrence after preperitoneal mesh repair. Traditional and laparoscopic. Ann Surg 225: 422-431.

8. Schmedt CG, Sauerland S, Bittner R (2005) Comparison of endoscopic procedures vs. Lichtenstein and other open mesh techniques for inguinal hernia repair: a meta-analysis of randomized controlled trials. Surg Endosc 19: 188-199.

9. Bittner R, Arregui ME, Bisgaard T, Dudai M, Ferzli GS, et al. (2011) Guidelines for laparoscopic (TAPP) and endoscopic (TEP) treatment of inguinal Hernia [International Endohernia Society (IEHS)]. Surg Endosc 25: 2773-2843.

10. Burcharth J (2014) The epidemiology and risk factors for recurrence after inguinal hernia surgery. Dan Med J 61: 1-17. 
11. Liew W, Wai YY, Kosai NR, Gendeh HS (2017) Tackers versus glue mesh fixation: an objective assessment of postoperative acute and chronic pain using inflammatory markers. Hernia 21: 549-554.

12. Kaul A, Hutfless S, Le H, Hamed SA, Tymitz K, et al. (2012) Staple versus fibrin glue fixation in laparoscopic total extraperitoneal repair of inguinal hernia: A systematic review and meta-analysis. Surg Endosc 26: 1269-1278.

13. Schjøth-Iversen L, Refsum A, Brudvik KW (2017) Factors associated with hernia recurrence after laparoscopic total extraperitoneal repair for inguinal hernia: a 2-year prospective cohort study. Hernia 21: 729-735.

14. Claus CMP, Rocha GM, Campos ACL, Bonin EA, Dimbarre D, et al. (2016) Prospective, randomized and controlled study of mesh displacement after laparoscopic inguinal repair: fixation versus no fixation of mesh. Surg Endosc 30: 1134-1140.
15. Garg P, Nair S, Shereef M, Thakur JD, Nain N, et al. (2011) Mesh fixation compared to non-fixation in total extraperitoneal inguinal hernia repair: A randomized controlled trial in a rural center in India. Surg Endosc 25: 3300-3306.

16. Mayer $F$, Niebuhr $H$, Lechner $M$, Dinnewitzer $A$, Köhler $G$, et al. (2016) When is mesh fixation in TAPP-repair of primary inguinal hernia repair necessary? The register-based analysis of 11,230 cases. Surg Endosc 30: 4363-4371.

17. Miserez M, Peeters E, Aufenacker T, Bouillot JL, Campanelli G, et al. (2014) Update with level 1 studies of the European Hernia Society guidelines on the treatment of inguinal hernia in adult patients. Hernia 18: 151-163. 